Revista de Comunicación y Salud, 2021, Vol. 11, 55-76

Editado por Cátedra de Comunicación y Salud

ISSN: 2173-1675

\title{
REDES SOCIALES Y LA ADICCIÓN AL LIKE DE LA GENERACIÓN Z
}

\author{
Social Media and the Like Addiction of Generation Z \\ Davinia Martín Critikián': \\ Universidad CEU San Pablo. España. \\ davinia.martincritikian@ceu.es \\ Marta Medina Núñez: \\ Universidad CEU San Pablo. España. \\ marta.medinanunez@ceu.es
}

\section{Cómo citar el artículo}

Martín Critikián, D. y Medina Núñez, M. (2021). Redes sociales y la adicción al like de la generación z. Revista de Comunicación y Salud, 11, 55-76. https://doi.org/10.35669/rcys.2021.11.e281

\begin{abstract}
Resumen
Psicólogos y expertos del mundo de la tecnología vienen advirtiendo, desde hace tiempo, que el uso frecuente y continuado de las redes sociales puede resultar en una adicción, lo que, a su vez, podría derivar en ansiedad, dependencia emocional, pérdida de motivación, falta de autocontrol e irritabilidad. Un estudio realizado por la Chicago Booth School of Business (2012) señalaba que las redes sociales tienen una capacidad de adicción mayor incluso que la del tabaco porque, entre otros motivos, acceder a estas plataformas resulta sencillo y, además, gratuito. Con el presente estudio, planteamos como objetivo principal analizar la importancia e impacto que supone el like en la generación Z, así como estudiar el contexto social de las redes sociales e investigar si el like afecta a esta generación y de qué manera. Para la elaboración de esta investigación se ha realizado, por un lado, una revisión bibliográfica sobre las redes sociales y el uso que los jóvenes hacen de ellas, identificando cuáles son sus favoritas, cuánto tiempo le
\end{abstract}

\footnotetext{
${ }^{1}$ Davinia Martín Critikián: Doctora en comunicación, y profesor ayudante doctor de 2011 a 2017 en la Universidad Internacional La Rioja y del 2017 a la actualidad en la Universidad CEU San Pablo. Líneas de investigación centradas en tendencias de comunicación, comportamiento del consumidor y RR.SS.
} 
dedican y las implicaciones que estas tienen sobre el cerebro humano. Por otro lado, se ha realizado un estudio de campo que se ha llevado a cabo en España a una muestra de 500 jóvenes de 18 a 26 años (los correspondientes a la generación Z), con el objetivo de conocer el uso que hacen de las redes sociales y analizar el impacto que tienen los likes sobre ellos. Entre las conclusiones principales destacamos un uso de redes sociales de más de tres horas diarias, siendo Instagram y WhatsApp las redes favoritas por esta generación. Si bien algunos miembros de este grupo social afirman sentirse inseguros, enfadados e incluso tristes si no reciben la respuesta esperada (traducida en likes o comentarios pospublicación) no modifican ni adaptan el tipo de contenido que comparten públicamente en base al número de likes que puedan conseguir. Finalmente, a pesar de que dicen no darle demasiada importancia a la retroalimentación recibida, suelen activar sus notificaciones en las redes sociales para estar a la última de posibles interacciones.

Palabras clave: comunicación, redes sociales, adicción, generación Z, internet.

\begin{abstract}
Psychologists and technology experts from all over the world have long warned that the frequent and continuous use of social networks can result in addiction, which, in turn, can lead to anxiety, emotional dependence, loss of motivation, lack of self-control and irritability. A study carried out by the Chicago Booth School of Business (2012) pointed out that social networks have a greater addiction capacity even than tobacco because, among other reasons, accessing these platforms is simple and, moreover, free. With the present study, we propose to analyze the importance and impact of 'likes' on generation $Z$, as well as to study the social context of social networks; we aim to investigate if these 'likes' affect generation Z and in what way. A bibliographic review on social networks and the use that young people make of them has been carried out, identifying which are their favorites, how much time they dedicate to them, what activities they carry out and how that affects the human brain. Also, a field study has been carried out in Spain with a sample of 500 young people between the ages of 18 and 26 (those corresponding to generation Z), with the aim of understanding the use they make of social networks and analyze the impact that 'likes' have on them. Among the main conclusions of this research, we found out that gen $Z$ uses social networks for more than three hours a day, with Instagram and WhatsApp being their favorite networks. Although some members of this social group claim to feel insecure, angry and even sad if they do not receive the expected response (translated into 'likes' or post-publication comments) they do not modify or adapt the type of content they share publicly based on the number of likes they can get. Finally, despite the fact that they say they do not give too much importance to the feedback received, they usually activate their notifications on social networks to be up to date with possible interactions.
\end{abstract}

Keywords: communication, social media, addiction, gen zers, internet. 
Redes sociales y la adicción al like de la generación Z

\section{INTRODUCCIÓN}

Las adicciones se caracterizan por dominar la voluntad de una persona acerca de una dependencia a una sustancia, relación o actividad. Según la Organización Mundial de la Salud (OMS), la adicción se considera una enfermedad cerebral, igual que otros trastornos neurológicos o psiquiátricos reconocidos. Esta es una enfermedad que afecta a la neurotransmisión y las interacciones dentro de la estructura de nuestro cerebro.

Al igual que otras enfermedades crónicas, la adicción se desarrolla, en ocasiones, con recaídas. Sin un tratamiento apropiado, la enfermedad es progresiva y puede concluir en discapacidad irreversible y/o muerte prematura. Es una enfermedad neurológica y necesita ser tratada por profesionales. Los pacientes adictos son enfermos recuperables, por lo que, con el correcto tratamiento, pueden rehabilitarse completamente (Fundación hay salida, 2020).

Las adicciones pueden llevarnos a problemas graves para nuestra salud tanto física como mental. Es importante detectarlas en un estado anticipado para así poder conseguir un diagnóstico y tratamiento efectivos. Asimismo, es muy importante la colaboración de amigos y familiares, tanto en la detección como en el tratamiento y posterior seguimiento. Esta dependencia puede llegar a causar daño a otras personas también, los que se encuentran en nuestro alrededor, ya que podemos hacer que ellos sufran de ansiedad o de estrés por preocupación, sin darnos cuenta de lo que estamos causando. Esta enfermedad puede surgir por muchas razones, siendo una de ellas el uso continuado de las redes sociales (Top Doctors, 2020).

\section{OBJETIVOS}

La presente investigación tiene como objetivo principal analizar la importancia e impacto que supone el like en la generación Z. Para la consecución de la misma, se proponen los siguientes objetivos específicos:

1. Estudiar el contexto social de las redes sociales.

2. Investigar si el like afecta a la generación $Z$ y de qué manera.

\section{METODOLOGÍA}

Para conducir la investigación de manera adecuada y lograr los objetivos planteados, se ha llevado a cabo, por un lado, una investigación documental haciendo uso de fuentes primarias y secundarias, analizando y consultando diferentes páginas web, libros de texto, revistas científicas, repositorios y tesis doctorales. Por otro lado, se ha realizado una investigación de tipo cuantitativo empleando como herramienta principal la encuesta. Para ello, se elaboró un cuestionario cerrado, con 33 preguntas, resultado de una combinación de cuestiones dicotómicas, politómicas y de múltiple respuesta, así como preguntas abiertas y semiabiertas. Con este cuestionario se realizó una encuesta online entre noviembre de 2020 y febrero de 2021, logrando una muestra de 500 participantes, 
Redes sociales y la adicción al like de la generación Z

entre 18 y 26 años de edad, reclutados fundamentalmente a través del correo electrónico, WhatsApp y avisos en las redes sociales.

\section{MARCO TEÓRICO}

\subsection{Uso de las redes sociales}

En el momento en que el uso del Smartphone impacta negativamente en el desarrollo de la vida cotidiana social, laboral, estudiantil o familiar se puede definir como "uso problemático del móvil" (cuyas consecuencias pueden ser: pérdida de tiempo, alteraciones de la conducta, de los ritmos del sueño, falta de control, aislamiento, bajo rendimiento, etc.) (Cuesta et al., 2020).

Los jóvenes, en la actualidad, tienen una dependencia a las redes sociales y a la opinión de los demás que puede llegar a ser perjudicial, intentando mostrar solo el lado bueno de las cosas en lugar de mostrarse como son. Los productos de entretenimiento que los jóvenes demandan se caracterizan por unas características que configuran nuevas formas de comunicación con las que se sienten más identificados, tales como la acción constante, la inmediatez, la posibilidad de hacer dos cosas a la vez junto a la portabilidad, la participación y la gran importancia de la imagen (Bernal, 2010).

Otra de las características de las nuevas generaciones es la necesidad de aceptación a través de las redes sociales; miembros de la generación $Z$ transmiten su vida con el fin de llamar la atención, camuflar la imagen con filtros y mostrar pequeñas partes de su vida. A priori, estas acciones parece que son símbolo de transparencia y accesibilidad, cuando, en realidad, se trata de todo lo contrario, esto libera en el cerebro humano dopamina, que al igual que con otras adicciones, es la que se encarga de nuestro sentimiento de placer o satisfacción. Como en todo, bien utilizada, la herramienta se convierte en algo fantástico; en tanto que, en el exceso, empiezan los problemas (Colombo, 2020).

Está demostrado que los procesos sociales en red aumentan la producción de dopamina un neurotransmisor que se produce en estados de placer y que abre los canales cognitivos, es así como se explica también las adicciones a la tecnología y la dependencia emocional de las redes sociales (Rodríguez, 2017).

No obtener la repercusión social que esperan es para ellos un fracaso, una frustración emocional que a veces tiene consecuencias graves por lo que ya existen estudios que muestran la necesidad del control de las emociones (Barrientos-Báez et al., 2020). Solo en Instagram, se dan más de 4.000 millones de likes al día. La necesidad de estar conectado o de mirar constantemente los likes puede convertirse en un pozo de vulnerabilidad del que será muy difícil salir porque nunca sacian a la persona, al contrario: siempre se quiere más (Catalán, 2020).

En definitiva, las nuevas tecnologías y las redes sociales están creando una dependencia psicológica y emocional sobre la que ya nos alertan expertos, médicos y psicólogos, aunque no todo es negativo en este empleo de las nuevas tecnologías ya que 
estas adicciones pueden ser detectadas y sobre todo combatidas, desde el empleo de las mismas (Sancho-Escrivá et al., 2020).

Lo digital está presente, pero sin una guía correcta, se pueden llegar a experimentar problemas psicológicos como la adicción a los likes, a vivir un mundo de fantasía en el cual se piensa que se puede ser perfecto, con un cuerpo acorde a ciertos patrones, condiciones económicas óptimas, fingir ser algo que no se es y ser aceptado por el resto, así como tener múltiples personalidades o incluso desarrollar capacidades digitales que en la vida de las personas no se alcanzan, todo por tratar de encajar. Esto trae añadido la facilidad de agresión o el bullying digital.

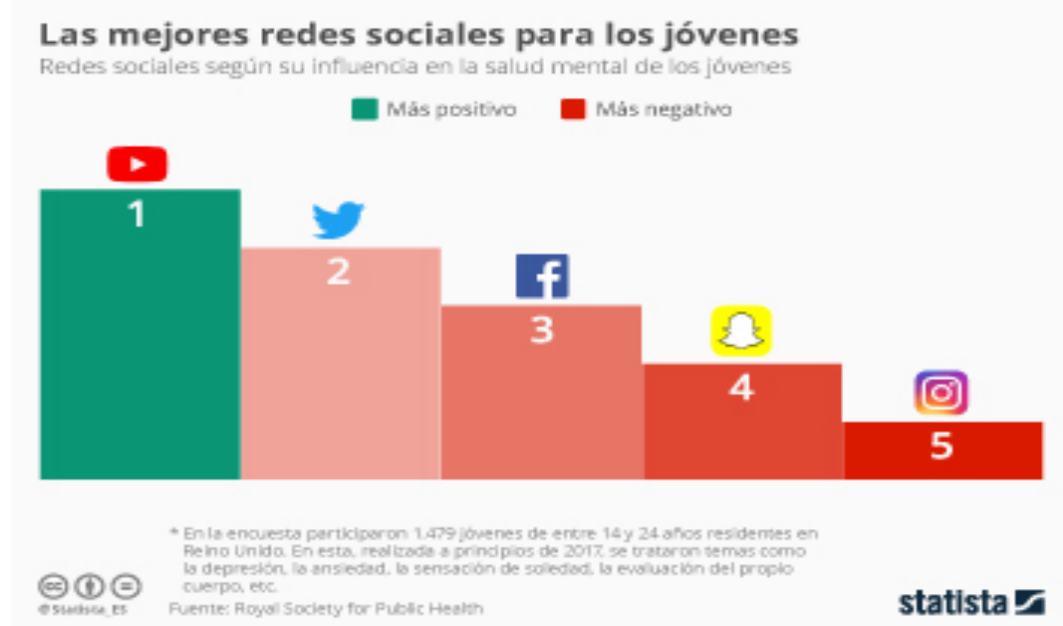

Figura 1. Las mejores redes sociales para los jóvenes

Fuente: Statista, 2020

La dependencia de las redes sociales se agrava con el uso permanente de estas herramientas; se calcula que vemos el teléfono móvil, por lo menos, unas 150 veces al día, en una interconexión permanente entre lo real y lo ficticio. En esta imagen, en la figura 1, podemos ver cómo el uso de las redes sociales puede suponer una influencia positiva o negativa, dependiendo de la plataforma que se trate.

Gracias a las redes sociales podemos entretenemos, interactuar, nos hacen sentir conectados, facilitan una comunicación aún más sencilla con la gente que conocemos, o incluso con desconocidos. Según Antoni Gutiérrez-Rubí:

Los millennials se consideran más independientes y críticos debido a la "autonomía" que les proporciona internet, a las posibilidades de acceso a fuentes de información con distintos puntos de vista y a las oportunidades de participación y debate que les ofrecen espacios como los foros o las redes sociales (Lago et al., 2016).

En la actualidad, si excluimos a la polivalente WhatsApp como red social, las más utilizadas son: Instagram, con un $68 \%$ de usuarios, YouTube, con casi un $80 \%$ de 
usuarios y Facebook, con un $70 \%$ de media (IAB, 2020). Algunas de esas redes sociales, como puede ser Instagram, permiten la opción de subir fotos y videos con diferentes efectos ya sea con filtros, colores retro, marcos, etc., lo que las hace unas aplicaciones muy intuitivas y fáciles de usar.

Facebook se posiciona como la red social más conocida en el mundo, la principal, la que más usuarios tiene, ya que se acerca a los 2 billones de usuarios activos en el momento. No obstante, el empleo de las redes sociales como Facebook presenta unas más amplias posibilidades que no escapan al mundo de lo mercantil, lo sostenible (Cervantes-Sintas, 2020; León León et al., 2018), la fidelización de grupos y un amplio etc., como la comunicación humanitaria y la salud (Arroyo-Almaraz et al., 2018) e incluso para difundir bulos sobre la misma (Piqueiras Conlledo et al., 2020). El objetivo de esta es compartir y producir contenido con la familia y/o amigos, incluso personas desconocidas. También, mediante esta se puede expandir o ampliar un negocio, es decir, darse a conocer para luego seguir adelante y ver el alcance que se llegaría a tener. Inclusive esta red social es muy buena para localizar eventos o buscar contactos (Gonçalves, 2016).

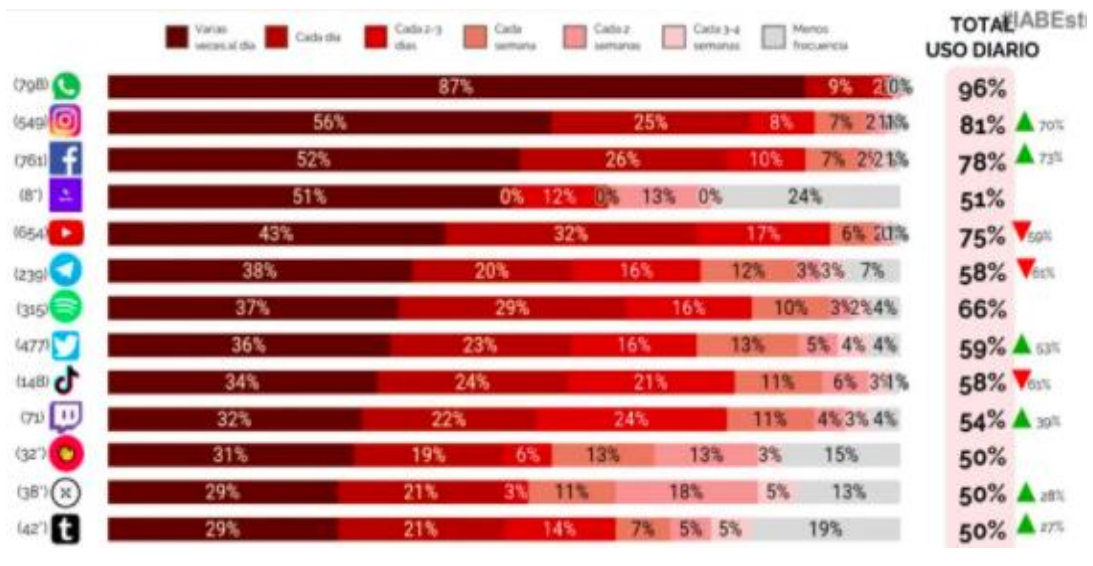

Figura 2. La frecuencia del uso de las redes sociales

Fuente: $I A B, 2020$

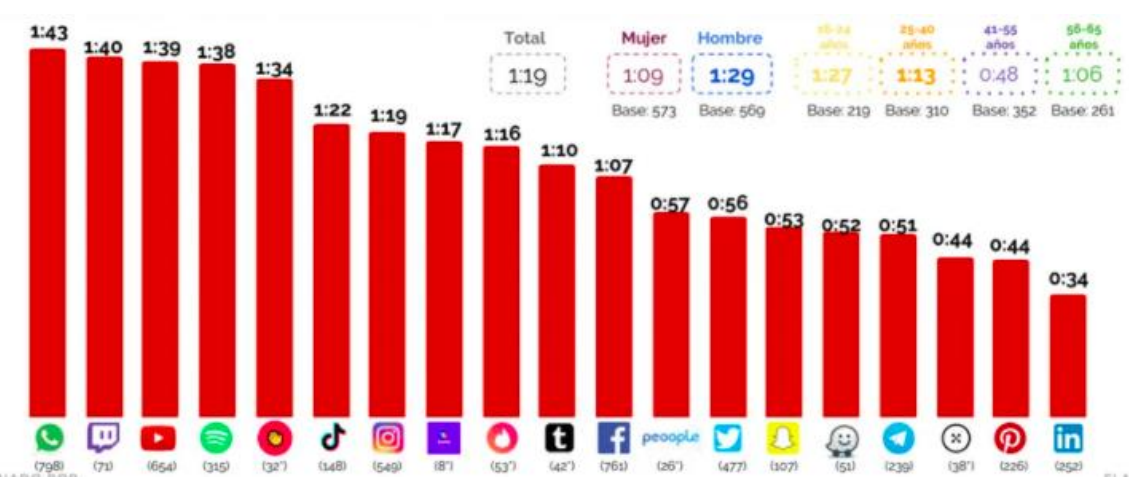

Figura 3. Intensidad del uso de las redes sociales

Fuente: IAB, 2020 
Según Claudia Martínez, la frecuencia y las horas al día de uso de las redes sociales ha aumentado significativamente debido a la pandemia mundial, esto se puede observar en el informe del IAB si comparamos el 2019 y el 2020 habiendo aumentado de promedio en 55 minutos. Encabezando la lista, encontramos WhatsApp, cuya función para mantener la salud en ciertos nichos está comenzando a ser estudiada (Barrientos-Báez et al., 2017), el estudio la incluye como red social en vez de como servicio de mensajería o llamadas instantáneas, seguido de Twitch, Youtube, Spotify, House Party o Tiktok, tal y como podemos apreciar en las figuras 2 y 3 esto contrasta con los datos del año anterior en los que redes sociales como Spotify, House Party o Tiktok, eran prácticamente inexistentes en cuanto al porcentaje de uso (Martínez, 2020).

\subsection{Perfil de las redes sociales}

Según el estudio del IAB (2020), en la actualidad, casi el 90\% de la población española utiliza las redes sociales y el rango de edad se encuentra entre los 16 a los 65 años de edad; aunque debido a la pandemia este porcentaje ha aumentado en el último año. Por otro lado, el perfil de usuario es muy similar entre ambos sexos (hombres $49 \%$ y mujeres $51 \%)$.

1. Uso, preferencias y valoración: Las redes que lideran el ranking de uso son: WhatsApp (85\%) que sigue posicionándose como la red favorita, Facebook (81\%) utilizado por un target más mayor, y Youtube $(70 \%)$ e Instagram (59\%) utilizado en gran parte por mujeres y por los más jóvenes. En quinto lugar, se encuentra Twitter, que mayoritariamente es utilizado por hombres. Sin embargo, la red que ha experimentado un gran crecimiento en comparación con las anteriores ha sido TikTok que ha pasado de un 3\% a un 16\% en un año. El target más joven (16-24), son los usuarios que utilizan mayor número de redes. En cuanto a la valoración, las aplicaciones mejor valoradas son: WhatsApp y Youtube $(8,4)$ así como Spotify $(8,2)$

2. Frecuencia de uso: WhatsApp sigue liderando el uso entre los usuarios con $96 \%$ que utiliza la aplicación a diario, seguida de Instagram con un $81 \%$ y Facebook con un $78 \%$. En cuanto a tiempo, los usuarios suelen pasar de media 1 hora y 43 minutos en WhatsApp, 1 hora y 39 minutos en Youtube, 1 hora y 38 minutos en Spotify y, por último, 1 hora y 34 minutos en House Party. Facebook ha bajado su frecuencia de uso durante el último año. En total, los usuarios dedican 1 hora y 19 minutos al día, siendo los hombres menores de 40 años quienes hacen un uso más intensivo de las redes sociales.

3. Actividades y acceso a RSS (Really Simple Syndication): Un estudio afirma que el $65 \%$ de los usuarios prefiere un contenido estable en comparación con uno fugaz, es decir, los consumidores han pasado de un snack content a un contenido más duradero y permanente. Los dispositivos más utilizados son: el teléfono móvil ( $97 \%$ prefieren este dispositivo antes que cualquier otro) y el ordenador, que se utiliza de manera más generalizada con un $93 \%$.

4. Relación con las marcas: Sin duda, las redes sociales han impulsado al aumento de ventas, ya que, gracias a estas plataformas, el consumidor está más actualizado en cuanto a las últimas tendencias, además de crear una relación más 
cercana entre cliente-empresa. Asimismo, las redes sociales suponen una forma de publicidad mucho más barata en comparación con anuncios en la televisión, en la radio... El banner o el formato gráfico es el formato publicitario preferido (53\%).

5. Generación Z vs Millennials: La generación Z utiliza con más frecuencia las redes sociales con una media de 5,4 cuentas. Estos usuarios prefieren el uso de redes como Instagram, seguida de WhatsApp y Youtube. En cambio, los millennials incorporan Facebook a sus redes más utilizadas (IAB, 2020). Además, los usuarios pertenecientes a la generación Z, según María Gabriela Palazzo, son más ansiosos, curiosos y esperan respuestas cada vez más rápidas en todos los ámbitos, indagan todo en Internet y no se sorprenden en absoluto con la expansión de las redes (Palazzo, 2014).

\subsection{Implicaciones de las redes sociales sobre el cerebro}

El empleo y uso habitual de las redes sociales tiene efectos sobre nuestro cerebro tal y como expusieron en la reunión 'Redes III', organizada por la compañía biomédica Pfizer el 19 de abril del 2016.

Desde el punto de vista de la biología, se ha demostrado que las redes sociales provocan cambios en los neurotransmisores como la oxitocina, la adrenalina, la dopamina, la serotonina, la testosterona y el cortisol. De esta manera, se activan los centros de recompensa y se incrementa la sensación de felicidad. Estos son los principales motivos por los que, cuando vemos productos en las redes sociales, se perciben mejor de lo que realmente son. La serotonina, por su parte, nos hacer modificar el comportamiento social a la hora de priorizar el individualismo frente al grupo social. Por otra parte, la testosterona la podríamos asociar con la capacidad de relacionarse y hacer nuevas amistades y, por último, el cortisol tendría un impacto en la fidelidad a las amistades.

Varios expertos neurólogos señalan la influencia de las redes sociales en temas como la disminución de capacidad de concentración o la pérdida de atención.

Algunos efectos positivos, en cambio, serían los nuevos métodos de aprendizaje creados gracias a las conexiones neuronales que se han adaptado al uso de estas redes sociales, lo cual confirma de la plasticidad del cerebro humano. Pero en contrapartida, se dan importantes efectos negativos, como la posibilidad de causar dependencia, dado que parte del procesamiento cerebral de las redes sociales tiene lugar en los circuitos relacionados con las recompensas y su uso no controlado podría estar asociado a algunos trastornos psiquiátricos como las adicciones (Bermejo, 2016).

Acerca de los efectos de las redes sociales sobre el cerebro, el doctor Pedro Bermejo, neurólogo y presidente de la Asociación Española de Neuroeconomía, explica:

Ya se han comprobado sobre nativos digitales y se conoce que estos aprenden de un modo ligeramente diferente a los que no lo son. Por una parte, los nativos digitales son capaces de hacer varias tareas a la vez con mejor resultado y por 
otra son más rápidos buscando información para dar respuesta a preguntas concretas (Bermejo, 2016).

$Y$ añade: "se ha comprobado que tienen mayor dificultad para discernir entre las fuentes de información fiables y las que no lo son, dándole más importancia a la información que captan de sus amigos y conocidos, y menos a las páginas web oficiales y más confiables" (Bermejo, 2016).

Durante esta reunión también se han abordado temas como la manera en la que el uso de las pantallas táctiles de los teléfonos inteligentes cambia la forma en que pulgares y cerebro trabajan en conjunto y cómo el uso de Internet puede reactivar el cerebro adulto. El comité de expertos remarcó la problemática de hacer un uso excesivo de las redes sociales, puesto que se han llegado a vincular ciertas enfermedades inflamatorias 0 auditivas (Bermejo, 2016).

La conectividad continuada y las redes sociales llevan al usuario a hacer un uso impulsivo y compulsivo del dispositivo, un hábito de consumo que se convierte en una amenaza para la concentración y el auto control del uso de internet y de las redes (Franco y Barrio, 2015).

\subsection{La influencia del like}

Uno de los grandes problemas que las redes sociales están generando es que los jóvenes están tan sumergidos en estas plataformas que no ven más allá de lo que estas les ofrecen.

Hoy en día, los jóvenes dependen de los likes que puedan llegar a tener sus publicaciones. Además, están tan pendientes de mostrar sus vidas a través de una cámara para que sus seguidores vean lo que hacen en cada momento, que no disfrutan de la propia realidad, ni de lo que viven al momento.

Cada vez más se tiende a emplear las redes sociales como un escaparate de las cosas positivas de la vida de los usuarios, es por ello que en estas plataformas se muestra una realidad distorsionada, ya que la vida no consiste en una línea recta, sino que tiene sus idas y venidas y eso en parte es lo bonito de la vida.

Como se ha señalado antes, los más afectados son los jóvenes, pero realmente esto también afecta a los adultos, solo que las consecuencias en los primeros son más graves. Esto se debe a que los adolescentes están en un periodo donde, además de definir por completo su identidad, tienen la autoestima más baja que un adulto. Por eso, las críticas, los comentarios y el no tener tantos likes como se esperaban les puede afectar más (Quintanilla, 2020).

Lo más importante para no dejar que la vida de los usuarios dependa de los likes es priorizar que estos usen el sentido común. Hacer que hagan y publiquen lo que les guste y no se sientan obligados a hacer algo por la repercusión (el número de likes). Además, 
Redes sociales y la adicción al like de la generación Z

hay que hacerles ver que, si ellos hacen lo que les gusta, no importa que a otros les guste o no.

Una de las razones por las cuales la sociedad de hoy en día desea tener tanto like es porque, principalmente, en la actualidad lo que más importa es la imagen que las personas dan a otros y las respuestas que reciben de los demás, es decir, hoy en día se depende de las respuestas que vengan del exterior. El hecho de que los usuarios dependan del número de likes que reciban nos muestra que estos parten de una autoestima baja. Esto hace que ese número de likes influya a la hora de valorarse a sí mismos y dejarse influenciar por los demás (Quintanilla, 2020).

Según un estudio de María José Establés, en las redes sociales es común la economía de la atención con tácticas como apoyarse en un grupo de WhatsApp para ganar popularidad. Así, los amigos comparten la publicación en sus respectivas redes, pidiendo que sus seguidores se conviertan en followers de la cuenta del amigo o amiga, que comenten o que den like a dicha publicación. La importancia de los likes y comentarios es lo que lleva a recurrir a una estrategia de self-branding como es dirigirse directamente a los propios followers para promover la participación en su cuenta. Además, se destaca cómo los jóvenes conciben su identidad en las redes sociales, principalmente en Instagram, como una extensión de su identidad offline (Establés et al., 2019).

Cada vez hay menos limitaciones para poder mezclar las diferentes sustancias expresivas (sonidos, letras, imágenes...) que puedan configuran una narración o un discurso construido como plataforma para la participación, la conversación, la interacción. Nos encontramos en la cultura del remix, una cultura que, amparada por el desarrollo tecnológico, se presenta como una revolución social que busca una arquitectura de la colaboración y la complicidad entre individuos (Cuesta y Menéndez, 2009).

Para Arturo Pilco Mindiola, también psicólogo clínico, es muy fácil depender de las respuestas positivas que se reciben y que la felicidad tiene que ver con ello. Es una adicción difícil de superar. "Su dispositivo móvil es un medio de comunicación, pero en realidad está siendo rehén", dice. Por eso menciona que el primer paso para aprender a escapar es entender que la necesidad constante de ellos (los me gusta) suele ser síntoma de baja autoestima. "Si puedes aprender a aumentar tu autoestima, puedes romper las cadenas mentales y emocionales que te atan. Pero claro, tener una buena autoestima no es tarea fácil, pero tampoco imposible. Hay que trabajar duro y practicar los consejos y técnicas más eficaces", aconseja. Es importante destacar que no todos controlan por igual esta dependencia y el uso de las redes sociales no afecta a todos por igual (Mindola, 2019).

Instagram experimentó, durante unas semanas, una versión de la red social en la que los likes no apareciesen, es decir, que no fueran visibles para los seguidores. Según los resultados de estas pruebas, pronto se decidirá si implantar los cambios como la versión definitiva de la aplicación. Con esto se busca que los usuarios interactúen con los contenidos de manera más genuina, no guiados por el precedente que establecieron quienes lo vieron antes que ellos. También persigue lograr interacciones más complejas

Revista de Comunicación y Salud, 2021, Vol. 11, 55-76 
y que propicien la comunicación más allá de la mecánica repetitividad del double tap, scroll, double tap. Facebook, por otro lado, no da señales de querer avanzar en ese sentido, por el contrario, parece propiciar las "reacciones".

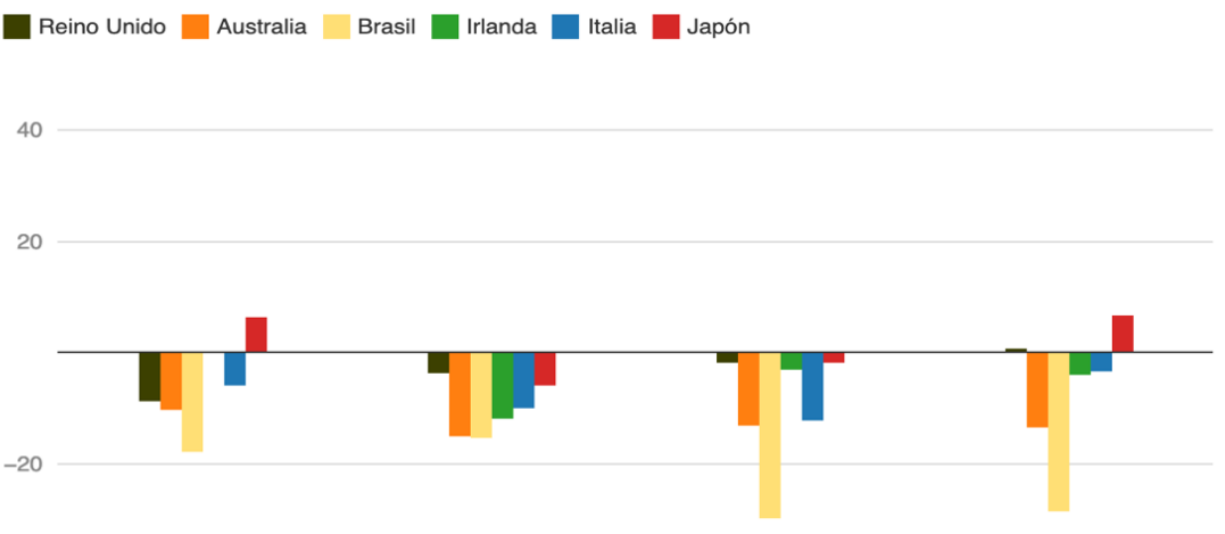

Figura 4. Cómo caen los likes si el like está oculto.

Fuente: El Confidencial

Tal y como podemos observar en la figura 4, otros países (aparte de España) han intentado ocultar el like en sus redes sociales. Podemos pensar que hay personas que dan like por una cuestión de aprobación, de sentirse parte de una comunidad y encajar en un grupo, en lugar de mostrarse tal y como son. Así lo ha demostrado un estudio elaborado por Hype Auditor. Esta empresa analizó las cuentas de 154.000 instagrammers famosos que reuniesen dos condiciones: uno, que cobrasen por publicitar marcas; $y$ dos, que al menos un $30 \%$ de sus seguidores procediesen de alguno de los países en los que Instagram había escondido el número de likes: Australia, Brasil, Irlanda, Italia y Japón.

Este estudio se dividió en cuatro niveles, en función del número de seguidores: entre 1.000 y 5.000 , entre 5.000 y 20.000 , entre 20.000 y 100.000 y entre 100.000 y un millón. En casi todos los casos, el resultado fue una caída estrepitosa. Llama especialmente la atención Brasil, donde los likes de los influencers que cuentan con una media entre 20.000 y 100.000 seguidores, cayeron un $29,63 \%$ y los de los que tienen entre 100.000 y un millón de seguidores, un $28,33 \%$. Solo hay una curiosa excepción: la de Japón, que aumentó tímidamente sus likes en dos de los cuatro grupos. Por lo demás, un fracaso generalizado (Otto, 2019).

Según Elliot Benjamin, filósofo en los campos de psicología humanista y transpersonal, en los últimos cuatro años, parece que la gente ha incrementado su adicción a las redes sociales. Ha dejado de estar en un primer plano la necesidad de ver y estar con gente, para aislarnos e interactuar a través de dispositivos electrónicos y redes sociales (Benjamin, 2020). 
La adicción a las redes es una variedad de adicción psicológica que afecta a nuestro comportamiento. Es como un impulso que no podemos controlar. Hay un mayor porcentaje de usuarios que se vuelve adicto y se involucra en uso excesivo o compulsivo de las redes. Las plataformas como Facebook, Instagram, Snapchat o TikTok producen los mismos circuitos neuronales que causan los juegos de azar e incluso las drogas para que los consumidores (los usuarios) sigan usándolas. Los estudios han demostrado que el flujo constante de retuits, me gusta y acciones de estos sitios han afectado al área de recompensa del cerebro para desencadenar el mismo tipo de reacción química que otras drogas (Huaytalla, 2016).

Uno de los principales impulsores de la adicción a las redes sociales es el FOMO (miedo a perderse algo, en español) que nos impulsa a hacer cosas incluso cuando no queremos hacerlas. Es igual a que cuando suena el teléfono, experimentas más el miedo a perderte algo, en caso de no cogerlo (Varchetta, 2020).

Las redes sociales aumentan nuestra autoestima. Tener nuestro propio espacio online significa que tenemos libertad absoluta para publicar sobre cualquier tema o sobre nosotros mismos. Es tu propio perfil y son tus propias reglas, no estás obligado a nada. Vemos todos los aspectos más destacados de la vida de las personas y de todas las cosas divertidas que están haciendo, nunca vemos el otro lado "detrás de la escena". Eso pasa porque la gente quiere verse bien ante los demás y las redes sociales te presentan esa oportunidad de tener una vida perfecta (Huaytalla, 2016). Este empleo de las redes presenta en nuestro caso una lectura más práctica en lo referente a la comunicación de la salud, tanto en detección como en pautas (Cofré-Soto, 2020).

\section{ESTUDIO DE CAMPO}

\subsection{Diseño}

Para el diseño de la presente investigación, que tiene como objetivo general analizar el impacto que supone el like en jóvenes de la generación Z, así como averiguar de qué manera les puede llegar a perjudicar a nivel mental y físico, se usaron las siguientes variables independientes:

- Edad (3 niveles): 18-20 años, 21-23 años y 24-26 años.

- Presencia en las redes sociales (2 niveles): sí, no

Por otro lado, las variables dependientes fueron:

- Redes sociales: preferencia de plataformas, número de perfiles activos, notificaciones.

- Modelo de uso: pasivo/activo, frecuencia de uso, tipo de actividad.

- Retroalimentación: engagement, uso del like y comentarios pospublicación. 


\subsection{Procedimiento}

El estudio se realizó en dos fases. Primero, se creó una encuesta de opinión, sincrónica, sobre un fenómeno particular: la adicción al like en usuarios de redes sociales de la generación $Z$. Se construyó un cuestionario cerrado, con 33 preguntas, resultado de una combinación de cuestiones dicotómicas, politómicas y de múltiple respuesta, así como preguntas abiertas y semiabiertas. Con este cuestionario se realizó una encuesta online entre noviembre de 2020 y febrero de 2021. Logramos una muestra de 500 participantes, entre 18 y 26 años de edad, reclutados fundamentalmente a través del correo electrónico, WhatsApp y avisos en las redes sociales.

La segunda fase del estudio consistió en la codificación, organización y análisis de datos, que se presentan a continuación.

\subsection{Resultados}

Tal y como se ha ido describiendo en los apartados anteriores, el objetivo de la encuesta realizada en esta investigación consiste en determinar la posible adicción al like que la generación $Z$ podría tener a las redes sociales. Otras variables que se han medido en la encuesta han sido: las redes sociales más usadas por este grupo social, el tiempo de conexión online, el contenido publicado, el uso activo y pasivo del like (dar a me gusta o recibir un me gusta) así como la activación de notificaciones en las redes sociales, entre otros.

La generación Z está formada por jóvenes cuya edad está comprendida, actualmente, entre los 11 y los 27 años de edad. De la muestra obtenida para este estudio (500 personas) más de la mitad (el 59\%) se encuentra entre los 18 y 20 años.

Una de las cuestiones objeto de estudio fue la presencia en las redes sociales y el consumo total que se hace de ellas. Para ello, se formularon preguntas como: ¿A qué Redes Sociales crees que dedicas más tiempo? ¿cuánto tiempo al día crees que le dedicas a las Redes Sociales? Ahora mira el tiempo de uso de las Redes Sociales en tu teléfono. ¿Cuánto tiempo pone que realmente pasas?

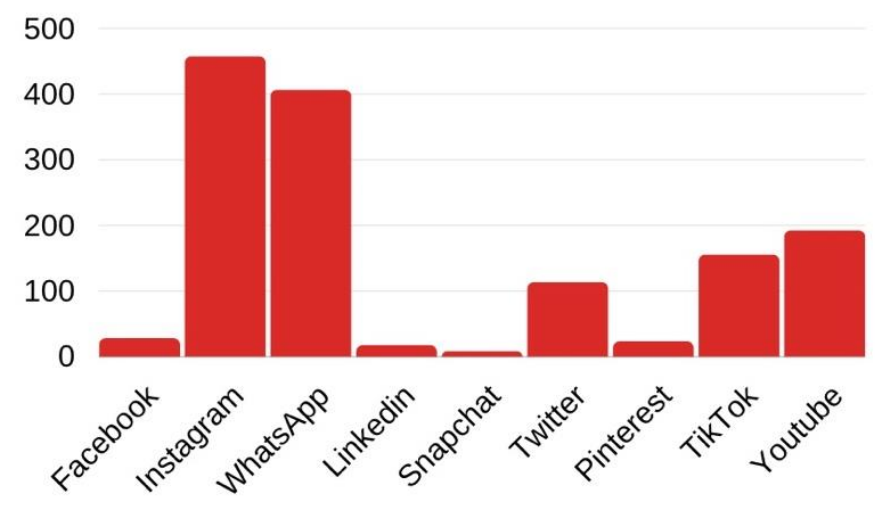

Revista de Comunicación y Salud, 2021, Vol. 11, 55-76 
Redes sociales y la adicción al like de la generación Z

Figura 5. Preferencia de redes sociales por la generación $Z$

Fuente. Elaboración propia (2021)

Un número muy elevado de encuestados (457 de 500) considera Instagram la plataforma social a la que dedican más tiempo, por detrás le sigue WhatsApp (406 encuestados) y como tercera opción, Youtube (192), Tiktok se sitúa en cuarta posición con 155 encuestados a favor. Por el contrario, las redes sociales a las que dicen dedicar menos tiempo y atención son: Snapchat (8), Linkedin (17), Pinterest (23) y Facebook (28). Estos datos coinciden, además, con El Estudio Anual De Redes Sociales del IAB (2020), que concluye que, si bien Facebook es la plataforma más conocida, no es la más usada entre el público usuario de redes sociales. Instagram, en cambio, sigue ganando relevancia y número de seguidores "sobre todo entre las mujeres y los más jóvenes" (Illa, 2020).

En lo relativo al tiempo de consumo de las redes sociales, en un primer momento, se les preguntó a los encuestados cuánto tiempo de media "creían" que dedicaban a las redes sociales. Un $40 \%$ afirmó hacer uso de estas plataformas durante más de tres horas diarias; un $35 \%$, entre dos y tres horas; un $22 \%$, entre 1 y 2 horas y un $3 \%$ menos de una hora al día. Estos datos coinciden, con un margen de error entre $2 \%$ y $3 \%$, con la respuesta que los encuestados nos dieron cuando les pedimos corroborar estos datos en sus teléfonos, comprobando el tiempo de uso medio de redes sociales que su dispositivo móvil había calculado a lo largo de la semana. Al 40\% inicial que afirmaba emplear más de 3 horas diarias en las redes sociales se unió un $2 \%$ más (42\%) y aquellos encuestados que decían pasar menos de una hora en estas plataformas (3\%), al comprobar su dispositivo móvil, este número aumentó en un $2 \%$ más (5\% total); sin embargo, aquellos que creían dedicarle entre 2 y 3 horas en las redes disminuyó en $1 \%(34 \%)$ y los que afirmaban pasar entre 1 y 2 horas al día cayeron en un 3\% (de un $22 \%$ a un $19 \%$ ). Con esto entendemos que, de manera general, la generación $Z$ es consciente del tiempo de uso que hace de estas plataformas, si bien en algún caso puntual puede que consideren que pasan más o menos tiempo en ellas.

Otro dato interesante: de todo el tiempo que dedican a las redes sociales, un $37 \%$ de los encuestados estima que pasa, de media, en cada red social entre 10 y 20 minutos (seguidos), mientras que un $27 \%$ opina que le dedica entre 20 minutos y media hora. Un $14 \%$ dice dedicar a una misma aplicación, en una sentada, entre media hora y 40 minutos, por el contrario, un $16 \%$ opina que no dedica más de 10 minutos a las redes sociales de forma continuada. 


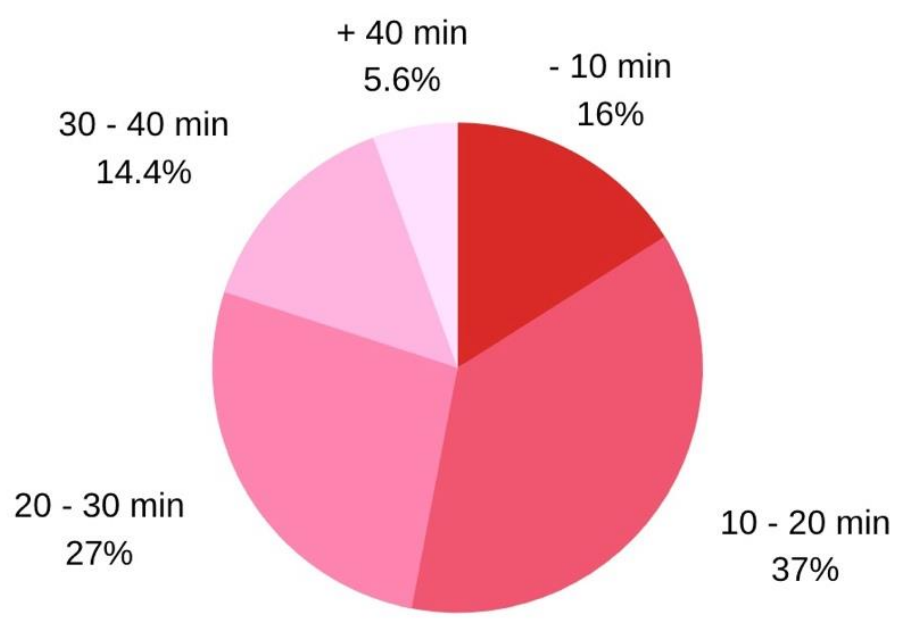

Figura 6. Tiempo de consumo de las Redes Sociales por la generación Z Fuente. Elaboración propia (2021)

Un $69 \%$ de encuestados afirma mirar y tener curiosidad por quién le ha dado un like a su publicación, sin embargo, un $28 \%$ dice no importarle demasiado. Estos datos quedan avalados cuando se comparan con la respuesta a la siguiente pregunta: " $¿$ Tienes activas las notificaciones en el móvil para que te avise cada vez que recibes alguna interacción en las redes sociales?" El $62 \%$ de la muestra dice tener "por lo general" las notificaciones activas, aunque depende, en ocasiones, de la red social, un 16\% opina necesitar siempre estas notificaciones y un $21 \%$ dice que no las activa, pues la retroalimentación pospublicación "puede esperar".

Cuando se les preguntó la frecuencia de conexión de las redes sociales en función a los likes recibidos por publicación "¿cada cuánto tiempo consultas si te han dado un "like" a tus fotos, vídeos, archivos...?" 163 encuestados afirmaron revisar la actualización de likes de forma esporádica y 129 incluso opinaron que "casi nunca" se vuelven a conectar por el hecho de revisar los números de likes de una publicación, 50 dijo hacerlo cada vez que abrían la aplicación, motivados por el posible like y solo 19 dijo chequear las redes "constantemente" en busca de esos "me gusta". Esta cuestión resulta relevante a la hora de determinar la adicción al like, objeto de este estudio, si bien no hay una respuesta dominante entre el grupo entrevistado, el mayor porcentaje de encuestados (32,6\%) considera que el like no es motivación suficiente para conectarse a las redes sociales, por el contrario, el $10 \%$ sí confiesa que los "me gusta" son la razón de comprobar este tipo de plataformas. 


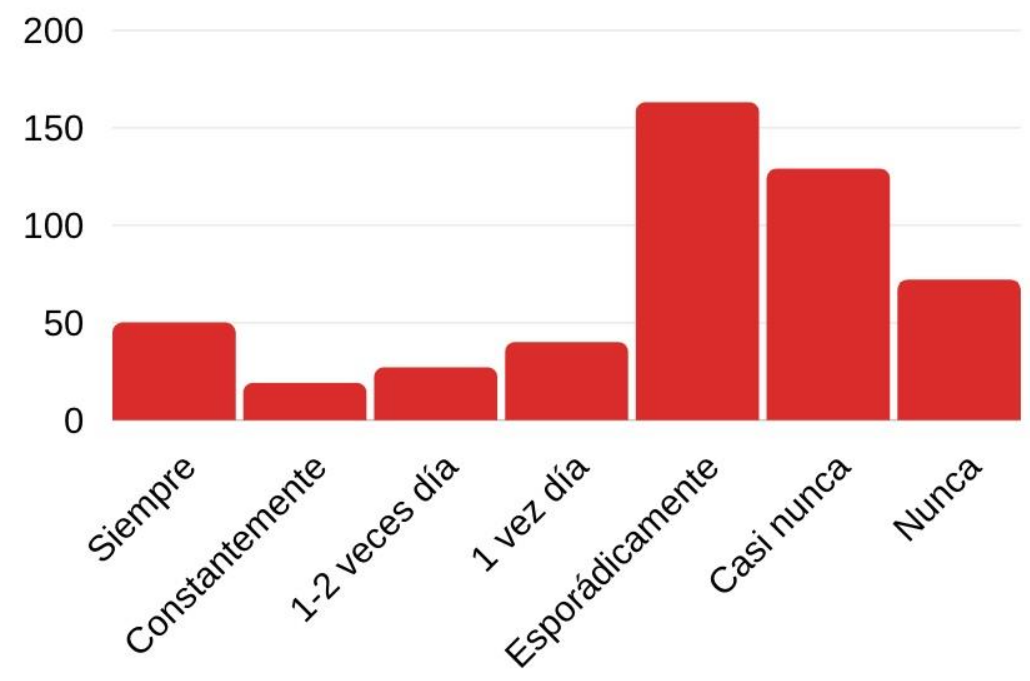

Figura 7. Comprobación de likes en redes sociales

Fuente. Elaboración propia (2021)

Llegados a este punto, consideramos oportuno descubrir de qué manera la generación $Z$ se siente afectada por la retroalimentación de sus publicaciones, es decir, cómo se sienten al publicar contenido online y de qué manera les afectan las reacciones o comentarios de sus seguidores. Ante la pregunta: "¿cómo te sientes si no dan un like a una foto que has publicado?" un $73 \%$ de encuestados (365 de 500) afirmaron que no les afectaba, de hecho "les daba un poco igual", el 13,2\% de ellos confesó sentirse inseguro y el $11,8 \%$ extrañado; una minoría, el $2,2 \%$, triste. Tan solo el $1 \%$ de encuestados dijo sentirse agobiado y enfadado. A pesar de los sentimientos negativos que esto pueda generar, el $70 \%$ de encuestados aseguró que no borraría una publicación aun habiendo recibido comentarios negativos o desagradables y un $90 \%$ afirmó que tampoco borraría una publicación si no recibe los "me gusta" esperados. 


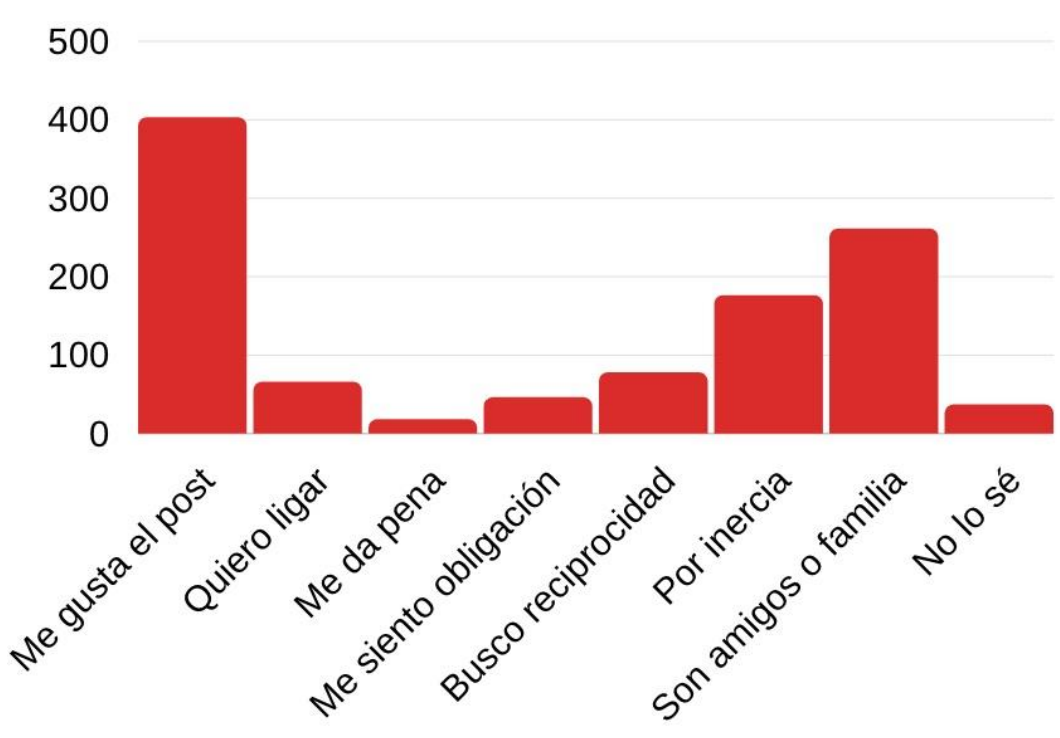

Figura 8. Motivos de dar like a publicaciones

Fuente. Elaboración propia (2021)

Cuando se les preguntó a los encuestados si alguna vez variaban el contenido que publicaban en función de los likes que pudieran potencialmente recibir, la respuesta fue prácticamente la misma: el $80 \%$ de ellos negó hacer publicaciones estratégicamente pensadas con el objetivo de aumentar sus likes.

Finalmente, del total de los encuestados, tan solo un $1 \%$ afirma dar likes a publicaciones aun cuando no le gusta el contenido y un 36 dice hacerlo "a menudo", por el contrario, un $54 \%$ que "casi nunca" comparte los "me gusta" si no es algo que realmente les llama la atención, agrada o atrae de alguna forma. Solo el $9 \%$ se muestra fiel a sus gustos y sentimientos a la hora de dar likes a publicaciones. Para indagar más sobre esta cuestión, se les preguntó a los encuestados el motivo de haber dado al like, aun cuando no les había agradado la publicación, 247 de 500 afirmó hacerlo por amistad o por familia (121) si bien 55 de ellos dijo hacerlo por compasión o no defraudar (42), 21 de los 500 confesó hacerlo "por caer bien".

\section{DISCUSIÓN}

Analizando los gráficos de las personas que utilizan las redes sociales diariamente, notamos cómo los jóvenes son los que más están involucrados en estas plataformas, siendo YouTube e Instagram las redes más usadas por adolescentes españoles. Estos datos, extraídos del estudio IAB (2020), coinciden con aquellos de nuestra investigación, donde una muestra de 500 jóvenes entre 18 y 26 años de edad (target más joven que utiliza mayor número de redes) afirma preferir Instagram, WhatsApp y Youtube por encima de otras redes sociales. 
Hoy en día, la adicción al like sigue creciendo, podemos tomarlo como algo positivo si es desde el punto de vista empresarial, ya que cuanto más tiempo pasen los usuarios en su página, mayor posibilidad de generar ingresos habrá; o negativo, si es desde el punto de vista del usuario que pierde el control sobre su tiempo sin ser consciente de ello. Siendo esto algo que nos afecta física y mentalmente deberíamos de empezar a controlarlo. En el marco teórico presentamos un dato sorprendente: comprobamos el teléfono móvil, por lo menos, unas 150 veces al día. En este tiempo, los miembros de la generación $Z$ dedican más de 3 horas diarias a las redes sociales, según nuestra investigación, y con una media de 10 a 20 minutos seguidos en la misma plataforma, un tiempo que podrían usar haciendo otras actividades más productivas. Según datos del IAB (2020), de este consumo, los usuarios suelen pasar de media 1 hora y 43 minutos en WhatsApp, 1 hora y 39 minutos en Youtube, 1 hora y 38 minutos en Spotify. Este hábito de consumo se puede convertir, de acuerdo con Franco y Barrio (2015) en una amenaza para la concentración y el auto control del uso de internet y de las redes.

De acuerdo con Palazzo (2014) los usuarios pertenecientes a la generación Z son más ansiosos, curiosos y esperan respuestas cada vez más rápidas en todos los ámbitos. De ahí se entiende la necesidad de activar notificaciones en las redes sociales para siempre estar a la última de los likes y comentarios recibidos. En este sentido, la generación Z, por lo general, dice tener las notificaciones activas, aunque depende, en ocasiones, de la red social. Una de las razones de permitir dichas notificaciones es la recepción, a tiempo real, de cualquier like o comentario que una publicación haya podido causar. Este grupo social confiesa revisar sus redes sociales de forma esporádica para comprobar si sus publicaciones han tenido una retroalimentación.

Ante el feedback recibido, la mayoría de la muestra objeto de estudio confiesa no sentirse afectada por no recibir likes, una minoría, en cambio, sí demuestra sentimientos de inseguridad, enfado, frustración e incluso tristeza. Estos datos coinciden con la opinión de Catalán (2020), quien advierte que la necesidad de estar conectado o de mirar constantemente estos likes puede convertirse en un pozo de vulnerabilidad del que será muy difícil salir. Además, de acuerdo con Quintanilla (2020), los jóvenes tienen la autoestima más baja que un adulto, por eso, las críticas, los comentarios y el no recibir tantos likes como les gustaría puede afectarles profundamente.

\section{CONCLUSIONES}

La generación Z prefiere Instagram por encima de otras redes sociales, a esta plataforma le sigue WhatsApp y, como tercera opción, Youtube; Tiktok se sitúa en cuarta posición. De media, este grupo social se considera un usuario pasivo de las redes, pues se dedica más a observar las publicaciones de otros que a compartir contenido propio.

El $40 \%$ de encuestados afirma hacer uso de estas plataformas durante más de tres horas al día; un $35 \%$, entre dos y tres horas; un $22 \%$, entre 1 y 2 horas y un $3 \%$ menos de una hora al día. De todo este tiempo, un $37 \%$ de la muestra estima que pasa, de media, en cada red social entre 10 y 20 minutos (seguidos), mientras que un $27 \%$ opina que le dedica entre 20 minutos y media hora. 
Con relación al objetivo principal de esta investigación "de qué manera los likes perjudican a la generacón Z" el $73 \%$ de encuestados afirma que no les afecta el hecho de recibir o no likes, pero un $13,2 \%$ sí confiesa sentirse inseguro y un $11,8 \%$ extrañado; una minoría, el 2,2\% se siente incluso triste. Tan solo el $1 \%$ de encuestados demuestran sentirse agobiados y enfadados. Aun así, el $80 \%$ niega hacer publicaciones estratégicamente pensadas con el objetivo de aumentar sus "me gusta". A pesar de estos sentimientos, estos jóvenes dicen no modificar ni adaptar el tipo de contenido que comparten públicamente en base al número de likes que puedan potencialmente conseguir.

Un $69 \%$ de encuestados afirma sentirse curioso por saber quién le ha dado un like a su publicación, sin embargo, un $28 \%$ dice no importarle demasiado. El $32,6 \%$ afirma revisar la actualización de los likes que recibe de forma esporádica y el 25,8\% opina que "casi nunca" se vuelven a conectar por el hecho de revisar los números de likes de una publicación; el 10\% dice hacerlo cada vez que abre cualquier aplicación, motivado por el un posible like. En este sentido, el $62 \%$ de la muestra dice tener "por lo general" las notificaciones activas, mientras que un $16 \%$ opina necesitar "siempre" estas notificaciones. Un $21 \%$ no las activa, pues no muestra interés por la retroalimentación pospublicación.

\section{REFERENCIAS}

Arroyo-Almaraz, I., Calle Mendoza, S. y Van Wyk C. (2018). La eficacia en la comunicación de las ONGD. El uso de Facebook en campañas de emergencia. Revista Latina de Comunicación Social, 73, 765-789. http://doi.org/10.4185/RLCS-2018-1281

Barrientos-Báez, A., Caldevilla-Domínguez, D. y García-García, E. (2017). APP para la tercera edad: utilidad, clases y valor social. Revista de Ciencias de la Comunicación e Información, 22(2), 1-11. http://doi.org/10.35742/rcci.2017.22(2).1-11

Barrientos-Báez, A., Caldevilla-Domínguez, D. y Martínez-González, J. A. (2021). Educación y gestión de emociones en Internet: hábitos de vida saludables. ESAMEC. Education Journal: Health, Environment and Citizenship.

Benjamin, E. Do We Live in a Social Media Technology Addicted Society? (s.f.). Integral World. Consultado el 27 de diciembre de 2020. http://www.integralworld.net/benjamin76.html

Bermejo, P. (2016, 19 de abril). Las redes sociales ya han modificado nuestro cerebro. Redes III reunión Pfizer. https://bit.ly/2QWjYA5

Bernal, A. I. (2010, 15 de septiembre). Definición conceptual de los medios de comunicación por un grupo de jóvenes españoles. El valor de internet. Revista Vivat Academia, 112, 22-32. https://doi.org/10.15178/va.2010.112.22-32 
Redes sociales y la adicción al like de la generación Z

Catalán, C. (2020, 1 de febrero). Esclavos de la dopamina. Navarra Capital. https://navarracapital.es/esclavos-de-la-dopamina/

Cervantes-Sintas, M. (2020). La comunicación desde el consejo de administración: estudio empírico sobre la legibilidad de los informes de sostenibilidad en español. Revista de Comunicación de la SEECI, 53, 103-134. https://doi.org/10.15198/seeci.2020.53.103-134

Cofré-Soto, E. (2020). Comunicación para la salud: aproximaciones epidemiológicas y socioculturales al cuerpo enfermo de las mujeres con cáncer de mama. Revista de Comunicación de la SEECl, 51, 169-183. http://doi.org/10.15198/seeci.2020.51.169$\underline{183}$

Colombo, D. Cómo funciona el cerebro cuando usas las redes sociales (s.f.). Cooperativa 93.3 FM. Consultado el 26 de diciembre de 2020. https://bit.ly/3fruNUG

Cuesta, U. y Cuesta, V. y Martínez, L. y Niño, J. I. (2020, 15 de enero). Smartphone: en comunicación, algo más que una adicción. Revista Latina de Comunicación Social, 75, 367-381.http://nuevaepoca.revistalatinacs.org/index.php/revista/article/view/15/10

Cuesta, U. y Menéndez, T. (2009). Prevención, comunicación y nuevas tecnologías: aspectos psicológicos entre jóvenes universitarios. Adicciones y nuevas tecnologías de la información y de la comunicación, pp. 84-85. Consultado el 29 de diciembre de 2020. https://bit.ly/3rBHTRY

Digital. (2012). ADDICTION: Study Finds Social Media to Be Like Smoking Cigarettes. https://bit.ly/3fp7SJP

Establés, M. J. y Guerrero, M. y Contreras, R. S. (2019, 11 de enero). Jugadores, escritores e influencers en redes sociales: procesos de profesionalización entre adolescentes. Revista Latina de Comunicación Social, 74, 214-236. http://www.revistalatinacs.org/074paper/1328/11es.html

Franco, R. y Barrio, M. G. (2015, 15 de noviembre). Infoxicación: implicaciones del fenómeno en la profesión periodística. Revista de Comunicación de La SEECI, 38, 141-181. https://doi.org/10.15198/seeci.2015.38.141-181

Fundación hay salida. ¿Qué es la adicción? (s.f.). Consultado el 27 de diciembre de 2020. https://www.fundacionhaysalida.com/que-es-la-adiccion/

Gonçalves, W. (2016, 1 de septiembre). Facebook: jtodo sobre la red social más usada en el mundo! Rock Content. https://rockcontent.com/es/blog/facebook/

Help adicciones. (2019, 12 de agosto). La adicción al like. Help adicciones: Centros especializados en trastornos adictivos. https://bit.ly/3cClCxT 
Redes sociales y la adicción al like de la generación Z

Huaytalla, C. (2016). Riesgo de adicción a redes sociales, autoestima y autocontrol en estudiantes de secundaria (E.P. de Psicología ed.). Revista Científica de Ciencias de la Salud, 9(1). https://revistas.upeu.edu.pe/index.php/rc salud/article/view/236/236

IAB Spain. (2020, 17 de junio). Presentación estudio redes sociales 2020. IAB Spain. https://iabspain.es/presentacion-estudio-redes-sociales-2020/

Illa, A. (2020, 17 de junio). Claves del Estudio de Redes Sociales IAB 2020 by Elogia. Elogia. https://bit.ly/3w7Fimd

Lago, D. y Direito, S. y Rodríguez, A. y López, X. (2016, 9 de noviembre). El consumo millennial de información política en televisión y redes sociales. Análisis de la campaña Elecciones Generales en España 2015. Revista Latina de Comunicación Social, 71, 1.151-1.169 http://www.revistalatinacs.org/071/paper/1139/59es.html

León León, M. E., Linares Herrera, M. P. y Junco Martínez, F. (2018). Oportunidades y desafíos relevantes de la educación para la comunicación ciudadana como concreción de una agenda de desarrollo local sostenible. Revista de Ciencias de la Comunicación e Información, 23(2), 15-25. http://doi.org/10.35742/rcci.2018.23(2).15-25

Martínez, C. (2020, 25 de agosto). Estadísticas del uso y percepción de las redes sociales en 2021. Cyberclick. https://bit.ly/3sDd0hc

Mas Delblanch, M. D. ISEP. (2021). Adicciones tecnológicas: internet, móviles y redes sociales. Blog de ISEP. Consultado el 10 de enero de 2021. https://bit.ly/3wfeCA0

Mindola, A. P. (2019, 28 de noviembre). Depender de likes: ¿Qué sentimos cuando no nos dan like? Extra. Ec. https://bit.ly/2Pmm1g3

Otto, C. (2019, 17 de noviembre). El imperio de los 'influencers' se desmorona: por qué su fortuna está en peligro. El Confidencial. https://bit.ly/2QTzAUQ

Palazzo, M. G. (2014, 20 de diciembre). Un recorrido por categorías y representaciones sobre juventud en las Ciencias Sociales. Revista Vivat Academia, 129, 119-147. https://doi.org/10.15178/va.2014.129.119-147

Piqueiras-Conlledo, P., De Marchis, G. y Cuesta Díaz, V. (2020). Análisis del contenido publicado en YouTube, Facebook e Internet sobre vacunas y anti vacunas. Revista de Comunicación y Salud, 10(1), 67-90. http://doi.org/10.35669/rcys.2020.10(1).67-90

Quintanilla, I. (2002). Psicología del consumido. PEARSON EDUCACIÓN, S. A., Madrid, 2002

Rodríguez, Y. M. (2017, 15 de marzo). Reconceptualización de la educación en la era digital: educominicacion, redes de aprendizaje y cerebro factores claves en los 
Redes sociales y la adicción al like de la generación Z

actuales escenarios de construcción de conocimiento. Revista de Comunicación de la SEECI, 42, 85-118. https://doi.org/10.15198/seeci.2017.42.85-118

Russell, J. (2017, 22 de junio). 5 Riesgos de seguridad en redes sociales y cómo evitarlos. Hootsuite. https://blog.hootsuite.com/es/riesgos-de-seguridad-en-redes-sociales/

Sancho-Escrivá, J. V., Fanjul Peyró, C., de la Iglesia Vayá, M, Montell, J. A. y Escartí Fabra, M. J. (2020). Aplicación de la inteligencia artificial con procesamiento del lenguaje natural para textos de investigación cualitativa en la relación médico-paciente con enfermedad mental mediante el uso de tecnologías móviles. Revista de Comunicación y Salud, 10(1), 19-41. http://doi.org/10.35669/rcys.2020.10(1).19-41

Top Doctors. Adicción. Top Doctors España. Consultado el 27 de diciembre de 2020). https://www.topdoctors.es/diccionario-medico/adiccion

Varchetta, M. Fraschetti, A. Mari, E. y Giannini, A. M. (2020). Adicción a redes sociales, Miedo a perderse experiencias (FOMO) y Vulnerabilidad en línea en estudiantes universitarios. Revista Digital de Investigación en Docencia Universitaria, 14(1), e1087. http://dx.doi.org/10.19083/ridu.2020.1187

\section{AUTORES}

\section{Davinia Martín Critikián}

Doctora en comunicación, y profesor ayudante doctor de 2011 al 2017 en la Universidad Internacional de La Rioja y del año 2017 a la actualidad en la Universidad CEU San Pablo. $\mathrm{Ha}$ centrado sus líneas de investigación en tendencias de comunicación, el comportamiento del consumidor y las redes sociales.

Orcid ID: http://orcid.org/0000-0002-6921-4707

\section{Marta Medina Núñez}

Doctoranda en Comunicación especializada, innovación y tecnologías por la Universidad CEU San Pablo, donde también trabaja como profesora colaboradora de Publicidad y RR.PP. Sus líneas de investigación están centradas en la comunicación social, la responsabilidad social corporativa y las redes sociales.

Orcid ID: http://orcid.org/0000-0003-1498-8083 